\title{
Matrix Bosonic realizations of a Lie colour algebra with three generators and five relations of Heisenberg Lie type
}

\author{
Gunnar SIGURDSSON ${ }^{a}$ and Sergei D. SILVESTROV ${ }^{b}$ \\ ${ }^{a}$ Department of Theoretical Physics, School of Engineering Sciences, Royal Institute of \\ Technology (KTH) - AlbaNova University Center, SE-106 91 Stockholm, Sweden \\ ${ }^{b}$ Centre for Mathematical Sciences, Lund University, P.O. Box 118, SE-221 00 Lund, \\ Sweden \\ E-mail: gunnsi@kth.se,ssilvest@maths.lth.se
}

\begin{abstract}
We describe realizations of a Lie colour algebra with three generators and five relations by matrices of power series in noncommuting indeterminates satisfying Heisenberg's canonical commutation relation of quantum mechanics. The obtained formulas are used to construct new operator representations of this Lie colour algebra using canonical representation of the Heisenberg commutation relation and creation and annihilation operators of the quantum mechanical harmonic oscillator.
\end{abstract}

2000 MSC: 17B75, 16G99, 16S32, 34K99, 81S05

\section{Introduction}

The main object studied in this paper is the unital associative algebra with three generators $A_{1}, A_{2}$, and $A_{3}$ satisfying defining commutation relations

$$
\begin{aligned}
A_{1} A_{2}+A_{2} A_{1} & =A_{3} \\
A_{1} A_{3}+A_{3} A_{1} & =0 \\
A_{2} A_{3}-A_{3} A_{2} & =0 \\
A_{2}^{2} & =0 \\
A_{3}^{2} & =0
\end{aligned}
$$

The main goal is to show how $A_{1}, A_{2}$, and $A_{3}$ can be expressed, using elements $A$ and $B$, obeying Heisenberg's canonical commutation relation

$$
A B-B A=I
$$

The canonical representation of the commutation relation (1.6) is given by choosing $A$ as the usual differentiation operator and $B$ as multiplication by $x$ acting on differentiable functions of one real variable $x$, on polynomials in one variable, or on some other suitable linear space of functions invariant under these operators. In quantum mechanics, these operators, when considered on the Hilbert space of square integrable functions, are essentially the same as the canonical Heisenberg-Schrödinger observables of momentum and coordinate, differing just by a complex scaling factor. The Heisenberg canonical commutation relation (1.6) is also satisfied by the annihilation and creation operators in a quantum harmonic oscillator. 
Since the 1970s, generalized (colour) Lie algebras have been an object of constant interest in both mathematics and physics $[1,3,4,5,8,9,10,11,12,13,14,15,16,17,19,20$, $21,25,26,27,23]$. Description of representations of these algebras is an important and interesting general problem. It is well known that representations of three-dimensional Lie algebras play an important role in the representation theory of general Lie algebras and groups, both as test examples and building blocks. Similarly, one would expect the same to be true for three-dimensional Lie colour algebras and superalgebras with respect to general Lie colour algebras and superalgebras. The representations of nonisomorphic algebras have different structure. In [26, 27], three-dimensional Lie colour algebras are classified in terms of their structure constants, that is, in terms of commutation relations between generators. In $[11,17,23]$, quadratic central elements and involutions on these algebras are calculated. In $[16,25]$, Hilbert space $*$-representations are described for the graded analogues of the Lie algebra $\mathfrak{s l}(2, \mathbb{C})$ and of the Lie algebra of the group of plane motions, two of the nontrivial algebras from the classification. The classification of $*$-representations in $[16,25]$ is achieved, using the method of dynamical systems based on generalized Mackey imprimitivity systems.

The colour Heisenberg Lie algebra is another important nontrivial algebra in the classification of three-dimensional Lie colour algebras obtained in $[26,27]$. In the paper [24] we approached representations of this algebra in a totally different way than it was done in $[16,25]$. Namely, we studied those representations which can be obtained as power series in operator representations of Heisenberg's canonical commutation relations by first obtaining in general realizations of the colour Heisenberg Lie algebra generators in terms of power series in elements of an associative algebra obeying the Heisenberg's canonical commutation relations and then combining these realizations with canonical representations of Heisenberg's canonical commutation relations.

In this paper we extend these investigations of realizations via Heisenberg's canonical commutation relations to another colour Lie algebra with three generators and five relations. This algebra can be considered as another colour analogue of the Heisenberg Lie algebra. However, we show that a structure of this algebra is quite different from that for the algebra considered in [24] as far as realization via Heisenberg's canonical commutation relations is concerned. In Section 2 we show that, with a natural choice for $A_{1}$ as the first generator of the Heisenberg algebra corresponding to differentiation, there are no nonzero power series in Heisenberg generators which can be taken as $A_{2}$ and $A_{3}$ so that the three relations (1.1)-(1.3) are satisfied. In Lemma 2.2, we describe all such formal power series solutions $A_{2}$ and $A_{3}$ for the first two relations (1.1)-(1.2) as in [24]. In Theorem 2.4, we present all such formal power series solutions for $A_{2}$ and $A_{3}$ satisfying the three relations (1.1)-(1.3) showing that $A_{3}=0$ is the only possibility. Using this result we get in Corollary 2.7 that $A_{2}=A_{3}=0$ must hold for such realizations of the five relations (1.1)-(1.5). However, by considering $2 \times 2$ matrices with entries chosen as formal power series in the noncommuting indeterminates $A$ and $B$ satisfying Heisenberg's canonical commutation relations, we demonstrate how it is possible to construct nontrivial realizations of (1.1)-(1.5). We also construct concrete operator representations by applying this construction to the canonical representation of Heisenberg canonical commutation relations and to the simple quantum mechanical harmonic oscillator.

\section{Matrix power series realizations}

Throughout this article $\mathbb{C}$ denotes the field of complex numbers and $\mathbb{N}$ the set of nonnegative integers. By $\mathbb{C}[x]$ and $\mathbb{C}[[x]]$ we mean the ring of polynomials and formal power series over $\mathbb{C}$, respectively. 
Consider a set $\left\{A_{1}, A_{2}, A_{3}\right\}$ in some associative algebra over $\mathbb{C}$ with unit element $I$ satisfying commutation relations (1.1)-(1.5). From relation (1.1) we obtain $A_{1} A_{2} A_{3}+A_{2} A_{1} A_{3}=A_{3}^{2}$, and hence by (1.2) it follows that

$$
A_{1}\left(A_{2} A_{3}\right)-\left(A_{2} A_{3}\right) A_{1}=A_{3}^{2}
$$

Using only (1.2) and (1.3) we may conclude that $A_{3}^{2}$ commutes with both $A_{1}$ and $A_{2}$, that is, $\left[A_{1}, A_{3}^{2}\right]=\left[A_{2}, A_{3}^{2}\right]=0$. If merely (1.1) and (1.2) are satisfied and if $A_{3}^{2}=\alpha^{2} I$ for some nonzero $\alpha \in \mathbb{C}$, then by $(2.1)$ we have

$$
A_{1}\left(A_{2} A_{3}\right)-\left(A_{2} A_{3}\right) A_{1}=\alpha^{2} I
$$

Applying the famous Wintner-Wielandt theorem [18, 28, 29], we have that no elements in any unital normed algebra can satisfy the Heisenberg canonical commutation relation

$$
A B-B A=\lambda I, \quad \lambda \neq 0
$$

So, we obtain from (2.2) the following result.

Proposition 2.1. The commutation relations

$$
A_{1} A_{2}+A_{2} A_{1}=A_{3}, \quad A_{1} A_{3}+A_{3} A_{1}=0
$$

together with $A_{3}^{2}=\alpha^{2} I, \alpha \neq 0$, cannot be satisfied by bounded operators on a Hilbert space or even generally by elements in any unital normed algebra.

When computing with power series in noncommuting elements $A$ and $B$ of an associative unital algebra $\mathfrak{A}$, we use the usual addition and multiplication rules of the Magnus algebra of noncommutative formal power series in two indeterminates (see [2]). However, we assume that $A$ and $B$ are not free, but satisfy at least the Heisenberg commutation relation as elements in $\mathfrak{A}$. We denote the algebra that we are working with by $\mathcal{H}_{1}\langle\langle A, B ; \mathfrak{A}\rangle\rangle$. In addition to the subalgebra of $\mathfrak{A}$ generated by $A$ and $B$, consisting of noncommutative polynomials in $A$ and $B$, the algebra $\mathcal{H}_{1}\langle\langle A, B ; \mathfrak{A}\rangle\rangle$ may contain other elements which are infinite noncommutative power series in $A$ and $B$ not belonging to $\mathfrak{A}$. The problem of equality of two elements in $\mathcal{H}_{1}\langle\langle A, B ; \mathfrak{A}\rangle\rangle$ is a very complex matter in itself, deeply connected both to the properties of noncommutative power series and Heisenberg's relation and to the structure of the algebra $\mathfrak{A}$, and properties of $A$ and $B$ in $\mathfrak{A}$. We say that an element of $\mathcal{H}_{1}\langle\langle A, B ; \mathfrak{A}\rangle\rangle$ is in the $(B, A)$ normal form (resp., $(A, B)$-normal form) if it is a noncommutative power series built of only ordered monomials $\left\{B^{j} A^{k} \mid j, k \in \mathbb{N}\right\}$ (resp., $\left\{A^{j} B^{k} \mid j, k \in \mathbb{N}\right\}$ ). In order to be able to enjoy the equality properties in a similar way with formal power series as in the polynomial case, we assume throughout this article that two formal power series in $A$ and $B$, written in the $(B, A)$-normal form (resp., in the $(A, B)$-normal form), are equal if and only if their coefficients are the same and in particular such a series is zero if and only if all coefficients are zero. This important equality assumption is actually an assumption on $\mathcal{H}_{1}\langle\langle A, B ; \mathfrak{A}\rangle\rangle$, on the algebra $\mathfrak{A}$ as well as on $A$ and $B$ as elements in $\mathfrak{A}$. In the particular case of polynomials in $A$ and $B$, that is, for the subalgebra of $\mathfrak{A}$ generated by $A$ and $B$, the assumption yields the same property as in $\mathcal{H}_{1}(A, B)$, namely that $\left\{B^{j} A^{k} \mid j, k \in \mathbb{N}\right\}$ (resp., $\left\{A^{j} B^{k} \mid j, k \in \mathbb{N}\right\}$ ) are linearly independent as subsets of $\mathfrak{A}$.

With the assumption above we may claim the equality of two elements of $\mathcal{H}_{1}\langle\langle A, B ; \mathfrak{A}\rangle\rangle$ if they are equal to the same element in $(B, A)$-normal form (resp., in $(A, B)$-normal form). However, it is important to observe that $\mathcal{H}_{1}\langle\langle A, B ; \mathfrak{A}\rangle\rangle$ may well contain elements which 
cannot be represented on $(B, A)$-normal form, or $(A, B)$-normal form or even on either of them. In most of the statements in this article we will adhere to the $(B, A)$-normal forms and the corresponding equality assumptions. But, we will also comment and at some instances will formulate the corresponding results when instead the $(A, B)$-normal forms and corresponding equality of series is used. Which of these assumptions is used will be clear from the context. We refer to $[6,7]$ for further discussion on power series extensions of the Heisenberg algebra, Diamond lemma, and normal forms.

In the paper [24] we have proved the following useful result.

Lemma 2.2. Let $A_{1}=A$ and assume that $A_{2}$ and $A_{3}$ are elements of the algebra $\mathcal{H}_{1}\langle\langle A, B ; \mathfrak{A}\rangle\rangle$ written in the $(B, A)$-normal form, that is,

$$
A_{2}=\sum_{j=0}^{\infty} \sum_{k=0}^{\infty} a_{j k} B^{j} A^{k}, \quad A_{3}=\sum_{j=0}^{\infty} \sum_{k=0}^{\infty} \tilde{a}_{j k} B^{j} A^{k}, \quad a_{j k}, \tilde{a}_{j k} \in \mathbb{C}
$$

Then $A_{1}, A_{2}$, and $A_{3}$ satisfy the commutation relations

$$
A_{1} A_{2}+A_{2} A_{1}=A_{3}, \quad A_{1} A_{3}+A_{3} A_{1}=0
$$

if and only if

$$
A_{2}=T(B, A) V(A)+B T(B, A) W(A), \quad A_{3}=T(B, A) W(A)
$$

where $T(B, A)=\sum_{k=0}^{\infty} \frac{(-2)^{k}}{k !} B^{k} A^{k}$ and $V(A), W(A) \in \mathbb{C}[[A]]$.

Remark 2.3. It follows by this lemma that the commutation relations

$$
A_{1} A_{2}+A_{2} A_{1}=A_{3}, \quad A_{1} A_{3}+A_{3} A_{1}=0
$$

can be satisfied by polynomial $A_{2}$ and $A_{3}$ (finite sums) only if $A_{2}=A_{3}=0$.

Theorem 2.4. Suppose that $A_{1}, A_{2}$, and $A_{3}$ are elements of the algebra $\mathcal{H}_{1}\langle\langle A, B ; \mathfrak{A}\rangle\rangle$ such that $A_{1}=A$ and $A_{2}, A_{3}$ are formal power series in the $(B, A)$-normal form given as

$$
A_{2}=\sum_{j=0}^{\infty} \sum_{k=0}^{\infty} a_{j k} B^{j} A^{k}, \quad A_{3}=\sum_{j=0}^{\infty} \sum_{k=0}^{\infty} \tilde{a}_{j k} B^{j} A^{k}, \quad a_{j k}, \tilde{a}_{j k} \in \mathbb{C}
$$

Then $A_{1}, A_{2}$, and $A_{3}$ will satisfy the commutation relations

$$
A_{1} A_{2}+A_{2} A_{1}=A_{3}, \quad A_{1} A_{3}+A_{3} A_{1}=0, \quad A_{2} A_{3}-A_{3} A_{2}=0
$$

if and only if

$$
A_{2}=T(B, A) V(A), \quad A_{3}=0
$$

where $T(B, A)=\sum_{k=0}^{\infty} \frac{(-2)^{k}}{k !} B^{k} A^{k}$ and $V(A) \in \mathbb{C}[[A]]$.

Proof. By Lemma 2.2 we have, considering only the two anticommutation relations, a general solution of the form

$$
\begin{aligned}
& A_{2}=T(B, A) V(A)+B T(B, A) W(A) \\
& A_{3}=T(B, A) W(A)
\end{aligned}
$$


Applying the rules of Lemma 4 in [24] yields

$$
\begin{aligned}
& A_{2} A_{3}=V(-A) W(A)+B W(-A) W(A) \\
& A_{3} A_{2}=W(-A) V(A)-B W(-A) W(A)+W^{\prime}(-A) W(A)
\end{aligned}
$$

and hence

$$
\begin{aligned}
A_{2} A_{3}-A_{3} A_{2}= & V(-A) W(A)-W(-A) V(A)-W^{\prime}(-A) W(A) \\
& +2 B W(-A) W(A)=0
\end{aligned}
$$

which is a functional-differential equation for $V$ and $W$. Thus $A_{2} A_{3}-A_{3} A_{2}=0$ implies $2 B W(-A) W(A)=0$, which for a formal power series $W(A)$ yields $W(-A) W(A)=0$. The set of complex formal power series $\mathbb{C}[[x]]$ is an integral domain and hence $W(A)=0$. This means that $A_{2}=T(B, A) V(A)$ and $A_{3}=0$.

Remark 2.5. Note that in Theorem 2.4, $A_{1} A_{2}+A_{2} A_{1}=A_{3}=0$ is the only nontrivial relation left. The other two relations are trivially satisfied when $A_{3}=0$, independently of $A_{1}$ and $A_{2}$. So, under conditions of Theorem 2.4, one can say that

$$
A_{1} A_{2}+A_{2} A_{1}=A_{3}, \quad A_{1} A_{3}+A_{3} A_{1}=0, \quad A_{2} A_{3}-A_{3} A_{2}=0
$$

is equivalent to

$$
A_{1} A_{2}+A_{2} A_{1}=0, \quad A_{3}=0
$$

Remark 2.6. In Lemma 2.2 , the formal series $A_{2}$ and $A_{3}$ are expressed in the $(B, A)$-normal form. This is a natural ordering when we think of $A$ as the usual differentiation operator $\partial$ and $B$ as a multiplication operator $M$ acting on differentiable functions on the real line, given by $\partial f=f^{\prime}$ and $M f(t)=t f(t)$. In other situations, it may be more appropriate to consider the reversed order.

Taking $A_{2}$ and $A_{3}$ in Lemma 2.2 to be in the $(A, B)$-normal form, but keeping $A_{1}=A$, the general solution will be changed to the following form:

$$
A_{2}=V(A) U(A, B)-W(A) U(A, B) B \quad A_{3}=W(A) U(A, B)
$$

where

$$
U(A, B)=\sum_{k=0}^{\infty} \frac{2^{k}}{k !} A^{k} B^{k}
$$

If we consider a solution in the $(A, B)$-normal form satisfying the three relations in Theorem 2.4, a completely similar proof shows that also in this case $A_{2}=V(A) U(A, B)$ and $A_{3}=0$.

Corollary 2.7. Suppose that $A_{1}, A_{2}$, and $A_{3}$ are elements of $\mathcal{H}_{1}\langle\langle A, B ; \mathfrak{A}\rangle\rangle$ such that $A_{1}=A$, and $A_{2}, A_{3}$ are formal power series in the $(B, A)$-normal form given as

$$
A_{2}=\sum_{j=0}^{\infty} \sum_{k=0}^{\infty} a_{j k} B^{j} A^{k}, \quad A_{3}=\sum_{j=0}^{\infty} \sum_{k=0}^{\infty} \tilde{a}_{j k} B^{j} A^{k}, \quad a_{j k}, \tilde{a}_{j k} \in \mathbb{C}
$$

Then $A_{1}, A_{2}$, and $A_{3}$ satisfy

$$
A_{1} A_{2}+A_{2} A_{1}=A_{3}, \quad A_{1} A_{3}+A_{3} A_{1}=0, \quad A_{2} A_{3}-A_{3} A_{2}=0, \quad A_{2}^{2}=A_{3}^{2}=0
$$

if and only if $A_{2}=A_{3}=0$. 
Proof. By Theorem 2.4, we know that $A_{2}=T(B, A) V(A)$ and $A_{3}=0$. Since

$$
A_{2}^{2}=T(B, A) V(A) T(B, A) V(A)=T(B, A)^{2} V(-A) V(A)=V(-A) V(A)
$$

the relation $A_{2}^{2}=0$ holds only if $V(-A) V(A)=0$, which, for a power series $V(A)$, yields $V(A)=0$ and $A_{2}=0$.

Now let $A_{1}, A_{2}$, and $A_{3}$ be $2 \times 2$ matrices on the form

$$
A_{1}=\left(\begin{array}{cc}
A_{11} & A_{12} \\
A_{21} & A_{22}
\end{array}\right), \quad A_{2}=\left(\begin{array}{cc}
0 & L \\
0 & 0
\end{array}\right), \quad A_{3}=\left(\begin{array}{cc}
0 & M \\
0 & 0
\end{array}\right)
$$

where $A_{11}, A_{12}, A_{21}, A_{22}, L$, and $M$ are elements of some associative algebra or ring $\mathcal{A}$. Then

$$
\begin{aligned}
& A_{1} A_{2}+A_{2} A_{1}=\left(\begin{array}{cc}
0 & A_{11} L \\
0 & A_{21} L
\end{array}\right)+\left(\begin{array}{cc}
L A_{21} & L A_{22} \\
0 & 0
\end{array}\right)=\left(\begin{array}{cc}
L A_{21} & A_{11} L+L A_{22} \\
0 & A_{21} L
\end{array}\right) \\
& A_{1} A_{3}+A_{3} A_{1}=\left(\begin{array}{cc}
M A_{21} & A_{11} M+M A_{22} \\
0 & A_{21} M
\end{array}\right)
\end{aligned}
$$

Therefore, $A_{1} A_{2}+A_{2} A_{1}=A_{3}$ is equivalent to the conditions

$$
L A_{21}=A_{21} L=0, \quad A_{11} L+L A_{22}=M
$$

and similarly we have $A_{1} A_{3}+A_{3} A_{1}=0$ if and only if

$$
M A_{21}=A_{21} M=0, \quad A_{11} M+M A_{22}=0
$$

Remark 2.8. Observe that for any elements $L$ and $M$ in $\mathcal{A}$ the relations

$$
A_{2} A_{3}-A_{3} A_{2}=0, \quad A_{2}^{2}=0, \quad A_{3}^{2}=0
$$

are always satisfied, since moreover we have $A_{2} A_{3}=A_{3} A_{2}=0$. Thus, under the conditions (2.3) and (2.4), elements $A_{1}, A_{2}$, and $A_{3}$ satisfy commutation relations (1.1)-(1.5). This gives a method of construction for realizations of (1.1)-(1.5).

Let us assume that either $L$ or $M$ are left or right invertible. Then $A_{21}=0$, and hence $A_{1}$ is upper triangular, that is,

$$
A_{1}=\left(\begin{array}{cc}
A_{11} & A_{12} \\
0 & A_{22}
\end{array}\right)
$$

So, we have the following useful statement.

Lemma 2.9. Let $L$ and $M$ be elements of an associative algebra or ring $\mathcal{A}$, such that at least one of them is left or right invertible in $\mathcal{A}$. Then the elements

$$
A_{1}=\left(\begin{array}{ll}
A_{11} & A_{12} \\
A_{21} & A_{22}
\end{array}\right), \quad A_{2}=\left(\begin{array}{cc}
0 & L \\
0 & 0
\end{array}\right), \quad A_{3}=\left(\begin{array}{cc}
0 & M \\
0 & 0
\end{array}\right)
$$

of the algebra (ring) $\mathcal{M}_{2}(\mathcal{A})$ of $2 \times 2$ matrices over $\mathcal{A}$ satisfy the commutation relations

$$
A_{1} A_{2}+A_{2} A_{1}=A_{3}, \quad A_{1} A_{3}+A_{3} A_{1}=0
$$


if and only if

$$
A_{21}=0, \quad \text { i.e., } \quad A_{1}=\left(\begin{array}{cc}
A_{11} & A_{12} \\
0 & A_{22}
\end{array}\right)
$$

together with

$$
A_{11} L+L A_{22}=M, \quad A_{11} M+M A_{22}=0
$$

Given $A_{1}$ and $A_{2}$ of the form in (2.7) and (2.5), respectively, there exists an $M$ so that $A_{3}$ of the form in (2.5) satisfies (2.6) if and only if

$$
A_{11}^{2} L+2 A_{11} L A_{22}+L A_{22}^{2}=0
$$

Proof. The first part was proved before the statement. The last relation follows by eliminating $M$ using (2.8). On the other hand, if (2.9) holds, then $M=A_{11} L+L A_{22}$ satisfies (2.8).

Lemma 2.10. Let the matrices

$$
A_{1}=\left(\begin{array}{cc}
B_{1} & A_{12} \\
0 & B_{1}
\end{array}\right), \quad A_{2}=\left(\begin{array}{cc}
0 & B_{2} \\
0 & 0
\end{array}\right), \quad A_{3}=\left(\begin{array}{cc}
0 & B_{3} \\
0 & 0
\end{array}\right)
$$

be elements of $\mathcal{M}_{2}(\mathcal{A})$, the algebra (ring) of $2 \times 2$ matrices over an associative algebra (ring) $\mathcal{A}$, and assume $B_{1}, B_{2}, B_{3}, A_{12} \in \mathcal{A}$. Then

$$
A_{1} A_{2}+A_{2} A_{1}=A_{3}, \quad A_{1} A_{3}+A_{3} A_{1}=0
$$

are satisfied if and only if $B_{1}, B_{2}$, and $B_{3}$ satisfy

$$
B_{1} B_{2}+B_{2} B_{1}=B_{3}, \quad B_{1} B_{3}+B_{3} B_{1}=0
$$

Moreover, $A_{2}^{2}=A_{3}^{2}=0$ and $A_{2} A_{3}=A_{3} A_{2}=A_{2} A_{3}-v A_{3} A_{2}=0$, independently on $B_{2}, B_{3} \in \mathcal{A}$.

Proof. The proof follows from the following easily checked equalities:

$$
A_{1} A_{2}+A_{2} A_{1}=\left(\begin{array}{cc}
0 & B_{1} B_{2}+B_{2} B_{1} \\
0 & 0
\end{array}\right), \quad A_{1} A_{3}+A_{3} A_{1}=\left(\begin{array}{cc}
0 & B_{1} B_{3}+B_{3} B_{1} \\
0 & 0
\end{array}\right)
$$

By Lemma 2.2 we know that if $A B-B A=I$ for $A$ and $B$ in some unital associative algebra $\mathfrak{A}$, then

$$
B_{1}=A, \quad B_{2}=T(B, A) V(A)+B T(B, A) W(A), \quad B_{3}=T(B, A) W(A)
$$

satisfy commutation relations

$$
B_{1} B_{2}+B_{2} B_{1}=B_{3}, \quad B_{1} B_{3}+B_{3} B_{1}=0
$$

and also if $B_{1}=A$ and $B_{2}$ and $B_{3}$ are $(B, A)$-normally ordered power series in $A$ and $B$, then $B_{1}, B_{2}$, and $B_{3}$ satisfy (2.11) if and only if $B_{2}$ and $B_{3}$ are of the form (2.10). A similar result is obtained if we choose to write $B_{2}$ and $B_{3}$ in the $(A, B)$-normal form.

Note also that we can apply Lemma 2.2 and its version in the $(A, B)$-normal form on a new set of generators $\tilde{A}=B$ and $\tilde{B}=-A$ satisfying $\tilde{A} \tilde{B}-\tilde{B} \tilde{A}=I$. Observing that $T(-A, B)=U(A, B)$ and $U(B,-A)=T(B, A)$, the resulting general solutions are

$$
\begin{array}{lll}
B_{1}=B, & B_{2}=U(A, B) V(B)-A U(A, B) W(B), & B_{3}=U(A, B) W(B) \\
B_{1}=B, & B_{2}=V(B) T(B, A)+W(B) T(B, A) A, & B_{3}=W(B) T(B, A)
\end{array}
$$

Combining this with Lemma 2.10, we can construct a realization of (1.1)-(1.5) using $A$ and $B$ satisfying the Heisenberg commutation relation $A B-B A=I$. 
Theorem 2.11. Assume $A$ and $B$ are two elements of an associative unital algebra or a ring (with identity) $\mathfrak{A}$, satisfying the Heisenberg commutation relation $A B-B A=I$. Let $T(B, A)=\sum_{k=0}^{\infty} \frac{(-2)^{k}}{k !} B^{k} A^{k}$ and define the following $2 \times 2$ matrices:

$$
\begin{aligned}
& A_{1}=\left(\begin{array}{cc}
A & A_{12} \\
0 & A
\end{array}\right) \\
& A_{2}=\left(\begin{array}{cc}
0 & T(B, A) V(A)+B T(B, A) W(A) \\
0 & 0
\end{array}\right) \\
& A_{3}=\left(\begin{array}{cc}
0 & T(B, A) W(A) \\
0 & 0
\end{array}\right)
\end{aligned}
$$

where $A_{12} \in \mathcal{H}_{1}\langle\langle A, B ; \mathfrak{A}\rangle\rangle$ and $V(A), W(A) \in \mathbb{C}[[A]]$. Then $A_{1}, A_{2}$, and $A_{3}$ satisfy the commutation relations

$$
A_{1} A_{2}+A_{2} A_{1}=A_{3}, \quad A_{1} A_{3}+A_{3} A_{1}=0, \quad A_{2} A_{3}-A_{3} A_{2}=0, \quad A_{2}^{2}=A_{3}^{2}=0
$$

Moreover, $A_{2} A_{3}=A_{3} A_{2}=0$.

When the $(A, B)$-normal forms and the corresponding equality assumption in $\mathcal{H}_{1}\langle\langle A, B ; \mathfrak{A}\rangle\rangle$ is used, the following analogue of Theorem 2.11 holds.

Theorem 2.12. Assume $A$ and $B$ are two elements of an associative unital algebra or a ring (with identity) $\mathfrak{A}$, satisfying the Heisenberg commutation relation $A B-B A=I$. Let $U(A, B)=\sum_{k=0}^{\infty} \frac{2^{k}}{k !} A^{k} B^{k}$, and define the following $2 \times 2$ matrices:

$$
\begin{aligned}
A_{1} & =\left(\begin{array}{cc}
A & A_{12} \\
0 & A
\end{array}\right) \\
A_{2} & =\left(\begin{array}{cc}
0 & V(A) U(A, B)-W(A) U(A, B) B \\
0 & 0
\end{array}\right) \\
A_{3} & =\left(\begin{array}{cc}
0 & W(A) U(A, B) \\
0 & 0
\end{array}\right)
\end{aligned}
$$

where $A_{12} \in \mathcal{H}_{1}\langle\langle A, B ; \mathfrak{A}\rangle\rangle$ and $V(A), W(A) \in \mathbb{C}[[A]]$. Then $A_{1}, A_{2}$, and $A_{3}$ satisfy the commutation relations

$$
A_{1} A_{2}+A_{2} A_{1}=A_{3}, \quad A_{1} A_{3}+A_{3} A_{1}=0, \quad A_{2} A_{3}-A_{3} A_{2}=0, \quad A_{2}^{2}=A_{3}^{2}=0
$$

Moreover, $A_{2} A_{3}=A_{3} A_{2}=0$.

Example 2.13. Introduce the operators

$$
A=M: f(x) \longmapsto x f(x), \quad B=-\partial: f(x) \longmapsto-f^{\prime}(x)
$$

acting on $\mathbb{C}[x]$. These operators satisfy $A B-B A=I$. Note also that $U(A, B)=U(M,-\partial)=$ $T(M, \partial)$. Let $A_{12}: \mathbb{C}[x] \rightarrow \mathbb{C}[x]$ be any operator. Then $A_{1}, A_{2}$, and $A_{3}$ defined in Theorem 2.12 are realized on $\mathbb{C}[x]$ by the operators

$$
\begin{aligned}
C_{1} & =\left(\begin{array}{cc}
M & C_{12} \\
0 & M
\end{array}\right) \\
C_{2} & =\left(\begin{array}{cc}
0 & V(M) T(M, \partial)+W(M) T(M, \partial) \partial \\
0 & 0
\end{array}\right) \\
C_{3} & =\left(\begin{array}{cc}
0 & W(M) T(M, \partial) \\
0 & 0
\end{array}\right)
\end{aligned}
$$


acting on $\mathbb{C}[x] \oplus \mathbb{C}[x]=\left\{\left(\begin{array}{l}f(x) \\ g(x)\end{array}\right)\right\}$. By the same theorem they satisfy the five relations for any power series $V(M)$ and $W(M)$ in $M$. Note that here $T(M, \partial): f(x) \mapsto f(-x)$ is the parity operator (see [24]). For example, if $V(t)=1$ and $W(t)=t^{2}$, then

$$
\begin{aligned}
& A_{1}:\left(\begin{array}{c}
f(x) \\
g(x)
\end{array}\right) \longmapsto\left(\begin{array}{c}
x f(x)+A_{12} g(x) \\
x g(x)
\end{array}\right) \\
& A_{2}:\left(\begin{array}{c}
f(x) \\
g(x)
\end{array}\right) \longmapsto\left(\begin{array}{c}
g(-x)+x^{2} g^{\prime}(-x) \\
0
\end{array}\right) \\
& A_{3}:\left(\begin{array}{c}
f(x) \\
g(x)
\end{array}\right) \longmapsto\left(\begin{array}{c}
x^{2} g(-x) \\
0
\end{array}\right)
\end{aligned}
$$

Example 2.14. Referring to [22] and [24, Example 5], we consider a sequence of functions $\left\{\phi_{\nu}\right\}_{\nu=0}^{\infty}$ defined by

$$
\sqrt{\nu+1} \phi_{\nu+1}(x)=a^{*} \phi_{\nu}(x), \quad \nu \geq 0 ; \quad \phi_{0}(x)=\frac{1}{\sqrt{\sqrt{\pi} x_{0}}} \exp \left(-\frac{1}{2}\left(\frac{x}{x_{0}}\right)^{2}\right)
$$

where $a^{*}$ is the linear operator

$$
a^{*}=-\frac{x_{0}}{\sqrt{2}} \partial+\frac{1}{\sqrt{2} x_{0}} M
$$

defined for any positive real constant $x_{0}$. It follows that

$$
\begin{aligned}
\phi_{n}(x) & =\frac{1}{\sqrt{n !}}\left(a^{*}\right)^{n} \phi_{0}(x)=\frac{1}{\sqrt{n ! \sqrt{\pi} x_{0}}}\left(a^{*}\right)^{n} \exp \left(-\frac{1}{2}\left(\frac{x}{x_{0}}\right)^{2}\right) \\
& =\frac{1}{\sqrt{2^{n} n ! \sqrt{\pi} x_{0}}} \exp \left(-\frac{1}{2}\left(\frac{x}{x_{0}}\right)^{2}\right) H_{n}\left(\frac{x}{x_{0}}\right)
\end{aligned}
$$

for $n=0,1,2, \ldots$, where $H_{n}$ are the Hermite polynomials. Defining the linear operator $a$ as

$$
a=\frac{x_{0}}{\sqrt{2}} \partial+\frac{1}{\sqrt{2} x_{0}} M
$$

it can be shown that $a \phi_{0}=0$ and $a \phi_{\nu}=\sqrt{\nu} \phi_{\nu-1}$ for $\nu \geq 1$. Moreover,

$$
\left(a^{*}\right)^{k} a^{k} \phi_{\nu}= \begin{cases}\frac{\nu !}{(\nu-k) !} \phi_{\nu} & \text { if } k \leq \nu \\ 0 & \text { if } k>\nu\end{cases}
$$

for any $k \in \mathbb{N}$. The sequence of functions $\left\{\phi_{\nu}\right\}_{\nu=0}^{\infty}$ describes the energy eigenstates of the simple quantum mechanical harmonic oscillator. Consider now the two differential operators $a^{*}$ ("creation" operator) and $a$ ("annihilation" operator) defined on the linear space $\Omega=$ $\operatorname{linspan}_{\mathbb{C}}\left(\left\{\phi_{\nu}\right\}_{\nu=0}^{\infty}\right)$ consisting of all complex linear combinations of functions from the set of eigenfunctions $\left\{\phi_{\nu}\right\}_{\nu=0}^{\infty}$. Since now $a a^{*}-a^{*} a=1$, we can define $A$ and $B$ in Theorem 2.11 as $A=a$ and $B=a^{*}$ acting on $\Omega$. Suppose $A_{12}: \Omega \rightarrow \Omega$ is any operator. Then $A_{1}, A_{2}$, and 
$A_{3}$ introduced in Theorem 2.11 are realized as the operators

$$
\begin{aligned}
A_{1} & =\left(\begin{array}{cc}
a & A_{12} \\
0 & a
\end{array}\right) \\
A_{2} & =\left(\begin{array}{cc}
0 & T\left(a^{*}, a\right) V(a)+a^{*} T\left(a^{*}, a\right) W(a) \\
0 & 0
\end{array}\right) \\
A_{3} & =\left(\begin{array}{cc}
0 & T\left(a^{*}, a\right) W(a) \\
0 & 0
\end{array}\right)
\end{aligned}
$$

acting on $\Omega \oplus \Omega=\left\{\left(\begin{array}{c}f \\ g\end{array}\right) \mid f, g \in \Omega\right\}$.

Recall that $a \phi_{0}=0$ and $a \phi_{\nu}=\sqrt{\nu} \phi_{\nu-1}$ for $\nu \geq 1$. Hence

$$
\begin{array}{ll}
A_{1}\left(\begin{array}{c}
\phi_{0} \\
0
\end{array}\right)=\left(\begin{array}{l}
0 \\
0
\end{array}\right), \quad A_{1}\left(\begin{array}{c}
\phi_{\nu} \\
0
\end{array}\right)=\sqrt{\nu}\left(\begin{array}{c}
\phi_{\nu-1} \\
0
\end{array}\right), \quad \nu \geq 1 \\
A_{2}\left(\begin{array}{c}
\phi_{\nu} \\
0
\end{array}\right)=\left(\begin{array}{l}
0 \\
0
\end{array}\right), \quad A_{3}\left(\begin{array}{c}
\phi_{\nu} \\
0
\end{array}\right)=\left(\begin{array}{l}
0 \\
0
\end{array}\right), \quad \nu \geq 0
\end{array}
$$

Moreover, for $\nu \geq 1$,

$$
A_{1}\left(\begin{array}{c}
0 \\
\phi_{\nu}
\end{array}\right)=\sqrt{\nu}\left(\begin{array}{c}
0 \\
\phi_{\nu-1}
\end{array}\right)+\left(\begin{array}{cc}
A_{12} & 0 \\
0 & 0
\end{array}\right)\left(\begin{array}{c}
\phi_{\nu} \\
0
\end{array}\right)
$$

Now let $V(a)=\sum_{k=0}^{\infty} v_{k} a^{k}$ and $W(a)=\sum_{k=0}^{\infty} w_{k} a^{k}$, where $v_{k}, w_{k} \in \mathbb{C}$. Since

$$
a^{k} \phi_{\nu}=\sqrt{\frac{\nu !}{(\nu-k) !}} \phi_{\nu-k}, \quad 0 \leq k \leq \nu
$$

it readily follows that

$$
T\left(a^{*}, a\right) V(a) \phi_{\nu}=\sum_{i=0}^{\nu}(-1)^{i} v_{\nu-i} \sqrt{\frac{\nu !}{i !}} \phi_{i}
$$

keeping in mind that $T\left(a^{*}, a\right) \phi_{\nu}=(-1)^{\nu} \phi_{\nu}$ for all $\nu \geq 0$.

Similarly, using the fact that $a^{*} \phi_{i}=\sqrt{i+1} \phi_{i+1}$, one obtains

$$
a^{*} T\left(a^{*}, a\right) W(a) \phi_{\nu}=\sum_{i=0}^{\nu}(-1)^{i} w_{\nu-i} \sqrt{i+1} \sqrt{\frac{\nu !}{i !}} \phi_{i+1}
$$

This means that

$$
\begin{aligned}
& A_{2}\left(\begin{array}{c}
0 \\
\phi_{\nu}
\end{array}\right)=\sum_{i=0}^{\nu+1}(-1)^{i}\left(v_{\nu-i}-i w_{\nu-i+1}\right) \sqrt{\frac{\nu !}{i !}}\left(\begin{array}{c}
\phi_{i} \\
0
\end{array}\right) \\
& A_{3}\left(\begin{array}{c}
0 \\
\phi_{\nu}
\end{array}\right)=\sum_{i=0}^{\nu}(-1)^{i} w_{\nu-i} \sqrt{\frac{\nu !}{i !}}\left(\begin{array}{c}
\phi_{i} \\
0
\end{array}\right)
\end{aligned}
$$

In the first expression, we have to assume that $v_{-1}=0$. Introduce a basis $\left\{\Phi_{\nu}\right\}_{\nu=0}^{\infty}$ of $\Omega \oplus \Omega$ given by

$$
\Phi_{2 \nu}=\left(\begin{array}{c}
\phi_{\nu} \\
0
\end{array}\right), \quad \Phi_{2 \nu+1}=\left(\begin{array}{c}
0 \\
\phi_{\nu}
\end{array}\right), \quad \nu \geq 0
$$


For $\nu \geq 0$, it follows that

$$
A_{1} \Phi_{0}=A_{2} \Phi_{2 \nu}=A_{3} \Phi_{2 \nu}=0
$$

And, for $\nu \geq 1$,

$$
A_{1} \Phi_{2 \nu}=\sqrt{\nu} \Phi_{2 \nu-2}, \quad A_{1} \Phi_{2 \nu+1}=\sqrt{\nu} \Phi_{2 \nu-1}+\left(\begin{array}{cc}
A_{12} & 0 \\
0 & 0
\end{array}\right) \Phi_{2 \nu}
$$

Moreover, for all $\nu \geq 0$,

$$
A_{2} \Phi_{2 \nu+1}=\sum_{i=0}^{\nu+1}(-1)^{i}\left(v_{\nu-i}-i w_{\nu-i+1}\right) \sqrt{\frac{\nu !}{i !}} \Phi_{2 i}, \quad A_{3} \Phi_{2 \nu+1}=\sum_{i=0}^{\nu}(-1)^{i} w_{\nu-i} \sqrt{\frac{\nu !}{i !}} \Phi_{2 i}
$$

\section{Acknowledgements}

This work was supported by the Swedish Research Council, The Crafoord Foundation, The Royal Swedish Academy of Sciences, The Royal Physiographic Society in Lund, and The Swedish Foundation for International Cooperation in Research and Higher Education (STINT). The first author is grateful to the Centre for Mathematical Sciences, Lund University for hospitality and support during his visits in Lund.

\section{References}

[1] V. K. Agrawala. Invariants of generalized Lie algebras. Hadronic J., 4 (1981), 444-496.

[2] Y. Bahturin. Basic Structures of Modern Algebra. Math. Appl., 265. Kluwer Academic Publishers, Dordrecht, 1993.

[3] Y. A. Bahturin, A. A. Mikhalev, V. M. Petrogradsky, and M. V. Zaicev. Infinite Dimensional Lie Superalgebras. Walter de Gruyter, Berlin, 1992.

[4] R. Campoamor-Stursberg and M. Rausch de Traubenberg. Color Lie algebras and Lie algebras of order F. J. Gen. Lie Theory Appl., 3 (2009), 113-130.

[5] H. S. Green and P. D. Jarvis. Casimir invariants, characteristic identities and Young diagrams for Colour algebras and superalgebras. J. Math. Phys., 24 (1983), 1681-1687.

[6] L. Hellström. The diamond lemma for power series algebras. Doctoral Thesis, No. 23, Umeå University, 2002.

[7] L. Hellström and S. D. Silvestrov. Commuting Elements in q-Deformed Heisenberg Algebras. World Scientific Publishing, New Jersey, 2000.

[8] V. G. Kac. Lie Superalgebras. Adv. Math., 26 (1977), 8-96.

[9] R. Kleeman. Commutation factors on generalized Lie algebras. J. Math. Phys., 26 (1985), 24052412.

[10] A. K. Kwasniewski. Clifford- and Grassmann-like algebras - old and new. J. Math. Phys., 26 (1985), 2234-2238.

[11] H. Ljungqvist and S. D. Silvestrov. Involutions in three-dimensional coloured Lie algebras. Research Reports 6, Department of Mathematics, Umeå University, 1996.

[12] J. Lukierski, V. Rittenberg. Color-de Sitter and color-conformal superalgebras. Phys. Rev. D., 18 (1978), 385-389.

[13] W. Marcinek. Colour extensions of Lie algebras and superalgebras. Preprint 746, University of Wrocław, 1990.

[14] W. Marcinek. Generalized Lie algebras and related topics, 1, 2. Acta Univ. Wratislaviensis (Matematyka, Fizyka, Astronomia), 55 (1991), 3-52. 
[15] M. V. Mosolova. Functions of non-commuting operators that generate a graded Lie algebra. Mat. Zametki, 29 (1981), 34-45.

[16] V. L. Ostrovskii and S. D. Silvestrov. Representations of real forms of the graded analogue of a Lie algebra. Ukraïn. Mat. Zh., 44 (1992), 1518-1524 (Russian). Translation in Ukrainian Math. J., 44 (1992), 1395-1401.

[17] L. Persson, S. D. Silvestrov, and P. Strunk. Central elements of the second order in threedimensional generalised Lie algebras. Czech. J. Phys., 47 (1997), 99-106.

[18] C. R. Putnam. Commutation Properties of Hilbert Space Operators and Related Topics. SpringerVerlag, New York, 1967.

[19] V. Rittenberg and D. Wyler. Generalized Superalgebras. Nuclear Phys. B, 139 (1978), 189-202.

[20] M. Scheunert. Generalized Lie algebras. J. Math. Phys., 20 (1979), 712-720.

[21] M. Scheunert. Graded tensor calculus. J. Math. Phys., 24 (1983), 2658-2670.

[22] F. Schwabl. Quantum Mechanics. 2nd ed. Springer-Verlag, Berlin, 1995.

[23] G. Sigurdsson and S. D. Silvestrov. Canonical involutions in three-dimensional generalised Lie algebras. Czech. J. Phys., 50 (2000), 181-186.

[24] G. Sigurdsson and S. D. Silvestrov. Bosonic Realizations of the Colour Heisenberg Lie Algebra. J. Nonlinear Math. Phys., 13 (2006), 110-128.

[25] S. D. Silvestrov. Hilbert space representations of the graded analogue of the Lie algebra of the group of plane motions. Studia Math., 117 (1996), 195-203.

[26] S. D. Silvestrov. Representations of commutation relations. A dynamical systems approach. Hadronic J. Suppl., 11 (1996), 1-116.

[27] S. D. Silvestrov. On the classification of 3-dimensional coloured Lie algebras. In "Quantum Groups and Quantum Spaces". R. Budzyński, W. Pusz, and S. Zakrzewski, Eds. Banach Center Publ., 40. Polish Acad. Sci., Warsaw, 1997, 159-170.

[28] H. Wielandt. Über die Unbeschränktheit der Operatoren der Quantenmechanik. Math. Ann., 121 (1949), 21.

[29] A. Wintner. The unboundedness of quantum-mechanical matrices. Phys. Rev. (2), 71 (1947), 738-739.

Received January 15, 2009

Revised August 10, 2009 